\title{
Clinical efficacy of transcutaneous triamcinolone acetonide injection for upper eyelid retraction and swelling in patients with thyroid eye disease
}

This article was published in the following Dove Press journal: International Medical Case Reports Journal

\author{
Ai Kozaki' \\ Hiroshi Nakamura ${ }^{2}$ \\ Toshu Inoue' \\ 'Olympia Eye Hospital, Tokyo, Japan; \\ 2Ooimachi Nakamura Eye Clinic, \\ Tokyo, Japan
}

Correspondence: Ai Kozaki

Olympia Eye Hospital, 2-18-12 Jingumae

Shibuya-ku, Tokyo I50000I, Japan

Tel +8I 33746898 I

Fax +8I 337468830

Email kouzaki@olympia.net
Aim: To evaluate the efficacy of transcutaneous triamcinolone acetonide (TA) injection for the treatment of upper eyelid retraction and swelling in thyroid eye disease (TED) patients.

Patients and methods: This is a case series. Three euthyroid TED patients with features of both upper eyelid retraction and swelling were recruited. TED signs appeared within 6 months prior to treatment. Next, $0.5 \mathrm{~mL}$ of TA $(40 \mathrm{mg} / \mathrm{mL})$ was transcutaneously injected targeting the orbital fat around the levator palpebrae superioris (LPS) muscle. At each visit, eyelid retraction was evaluated by palpebral fissure height and the presence of scleral show above the superior corneoscleral limbus. Eyelid swelling was judged by the appearance of upper eyelid bulging and the lack of an eyelid sulcus. In addition, the LPS muscle, orbital and retro-orbicularis oculi fat were observed using MRI before and after treatment.

Results: Two patients had resolution of their upper lid retraction and swelling within 12 months. In the other patient who had proptosis, eyelid retraction resolved, while eyelid swelling remained mild. In all cases, MRI revealed that treatment resulted in thinner LPS muscle and reduced fat swelling. In addition, both high-intensity signals on T2 images and prolonged T2 relaxation time were normalized. No adverse signs were observed.

Conclusion: Transcutaneous TA injection was effective as a treatment for upper eyelid retraction and swelling in euthyroid TED patients who had signs within 6 months prior to treatment. Transcutaneous TA injection induced reduction of LPS muscle enlargement and fat tissue swelling. These reductions by transcutaneous TA injection were verified using MRI.

Keywords: thyroid eye disease, transcutaneous triamcinolone injection, upper eyelid retraction, eyelid swelling, MRI, ROOF, LPS muscle

\section{Introduction}

Upper eyelid retraction and swelling are frequent findings in patients with thyroid eye disease (TED). ${ }^{1,2}$ The incidences of eyelid retraction and swelling in TED patients are $58 \%-98 \%$ and $47 \%-75 \%$, respectively. ${ }^{2,3}$ TED is three to nine times more common in women, ${ }^{4}$ and patients' age with upper eyelid retraction and swelling is mostly between 20 and 50 years. $^{2}$ Therefore, the appearance of such retraction and swelling causes cosmetic discomfort. 5,6

Upper eyelid retraction usually widens the palpebral fissure ${ }^{7}$ and exposes the sclera between the limbus and eyelid margin (referred to as scleral show), ${ }^{8}$ while the upper eyelids normally cover the superior cornea at the 12 o'clock position. The eyelid retraction is caused by malfunction of muscles in the upper lid, primarily Mueller's ${ }^{9}$ and the levator palpebrae superioris (LPS) muscles. ${ }^{3,10}$ In terms of Mueller's muscle, it has 
been suggested that overactivity of the sympathetic nervous system, enhanced by excessive secretion of thyroid hormone due to hyperthyroidism, stimulates the muscle tone, resulting in lid retraction. ${ }^{9}$ Another mechanism could be inflammation of the LPS muscle, and inflammation of the LPS muscle is thought to be induced by antithyroid autoantibodies, that is, TSH receptor antibodies (TRAb) and thyroid stimulating antibodies (TSAb). ${ }^{11,12}$ During the early to middle active inflammatory phase, the LPS muscle becomes inflammatorily edematous, increasing the muscle width and restricting movement, resulting in eyelid retraction. In the late active phase, fibrosis arises, causing LPS muscle contracture and maintaining the upper eyelid retraction for a long period. For such eyelid retraction induced by inflammation, steroid therapy could be effective before the late active phase. ${ }^{13}$

The swelling of the upper eyelid is caused by enlargement of the LPS muscle and eyelid/orbital fat. ${ }^{14}$ Although there are no strict criteria for eyelid swelling, ${ }^{4}$ it is clinically defined by eyelid bulging and lack of an upper eyelid sulcus. In MRI, T1-weighted sagittal sections reveal that eyelid swelling is caused by LPS muscle enlargement and orbital fat swelling. ${ }^{10,14}$ In addition, the degree of inflammatory edema in the LPS muscle could be quantitatively estimated as prolonged T2 relaxation time and high-intensity areas in T2-weighted fat-suppression images. ${ }^{15,16}$

Clinically, eyelid retraction frequently causes keratoconjunctivitis sicca and superior limbic keratoconjunctivitis. ${ }^{17}$ The retraction and swelling of the upper eyelid often impacts quality of life ${ }^{6}$ due to its appearance. Various treatments have resolved these issues. An $\alpha$-adrenergic blocker ophthalmic solution is effective for hypertonia in Mueller's muscle, but not for inflammation of the LPS muscle. ${ }^{9}$ Botulinum toxin injection resolves upper eyelid retraction, but needs to be periodically applied. ${ }^{18}$ Steroid pulse therapy has been an effective treatment, but has some side effects, including diabetes, hyperlipidemia and so on. ${ }^{19}$ Surgical treatments have been performed; however, these treatments can occasionally have unexpected outcomes, including over-/undercorrection and the undesirable double eyelid line. ${ }^{20}$

Recently, it has been reported that subconjunctival injection of triamcinolone acetonide (TA) targeting Mueller's muscle is effective in reducing upper eyelid retraction in the early congestive stage of TED patients. ${ }^{21-24}$ Several reports suggested that subconjunctival TA injections resulted in an improvement of eyelid retraction in $68 \%-75 \%$ of patients from 1 to 4 times. ${ }^{22-24}$ In addition, it was reported that such injection reduces inflammatory enlargement of the LPS muscle $^{23}$ and upper eyelid swelling. ${ }^{24}$ However, subconjunctival
TA injection causes increased intraocular pressure ${ }^{22-24}$ and menstrual irregularities. ${ }^{23}$

Herein, we studied whether transcutaneous TA injection targeting the LPS muscle and surrounding orbital fat was effective in reducing upper eyelid retraction and swelling in euthyroid TED patients. Treatment was initiated within 6 months of the appearance of TED signs. Using clinical assessment and MRI, significant improvement in upper eyelid retraction and swelling was observed in these patients.

\section{Patients and methods}

Between January and June 2009, patients diagnosed with TED with both upper eyelid retraction and swelling, either unilaterally or bilaterally, were recruited. Thyroid function of all patients was normal with or without medication at their first visit. Treatment was initiated within 6 months of the appearance of TED signs.

To prepare for transcutaneous TA injection, the upper eyelid was cooled with an ice pack for 1 minute to minimize pain and subcutaneous bleeding. With patients assuming downward gaze in the supine position, the upper eyelid skin was pulled (Figure 1A, B). A 26-guage needle was inserted to a depth of $\sim 1 \mathrm{~cm}$, and after confirming the absence of blood reflux, $0.5 \mathrm{~mL}$ of TA $(40 \mathrm{mg} / \mathrm{mL})$ was injected targeting the orbital fat around LPS muscle (Figure 1C). TA was injected only once for each eye.

At each visit, upper eyelid retraction and swelling were clinically evaluated (Table 2). Upper eyelid retraction was evaluated by measuring the palpebral fissure height as well as the presence of scleral show. Eyelid swelling was assessed by the presence of eyelid bulging from the upper eyelid margin and the lack of an upper eyelid sulcus. In addition, the LPS muscle and orbital fat tissue were observed using MRI before and at 6 or 8 months after treatment. LPS muscle enlargement and fat swelling were observed in T1-weighted sagittal images, and inflammatory edema in the LPS muscle was quantitatively examined with $\mathrm{T} 2$ relaxation time and T2-weighted fat-suppression images. Upper eyelid swelling was evaluated by the orbital fat around LPS muscle and the retro-orbicularis oculi fat (ROOF) thickness with sagittal MRI. To assess the potential for TA-induced complications, ${ }^{22-24}$ intraocular pressure was measured before and at 1, 3, 6 and 12 months after treatment. Patients were also questioned about any other problems including menstrual disorders.

\section{Ethics statement}

Written informed consent was obtained from the patients for publication of these case reports and accompanying images. 


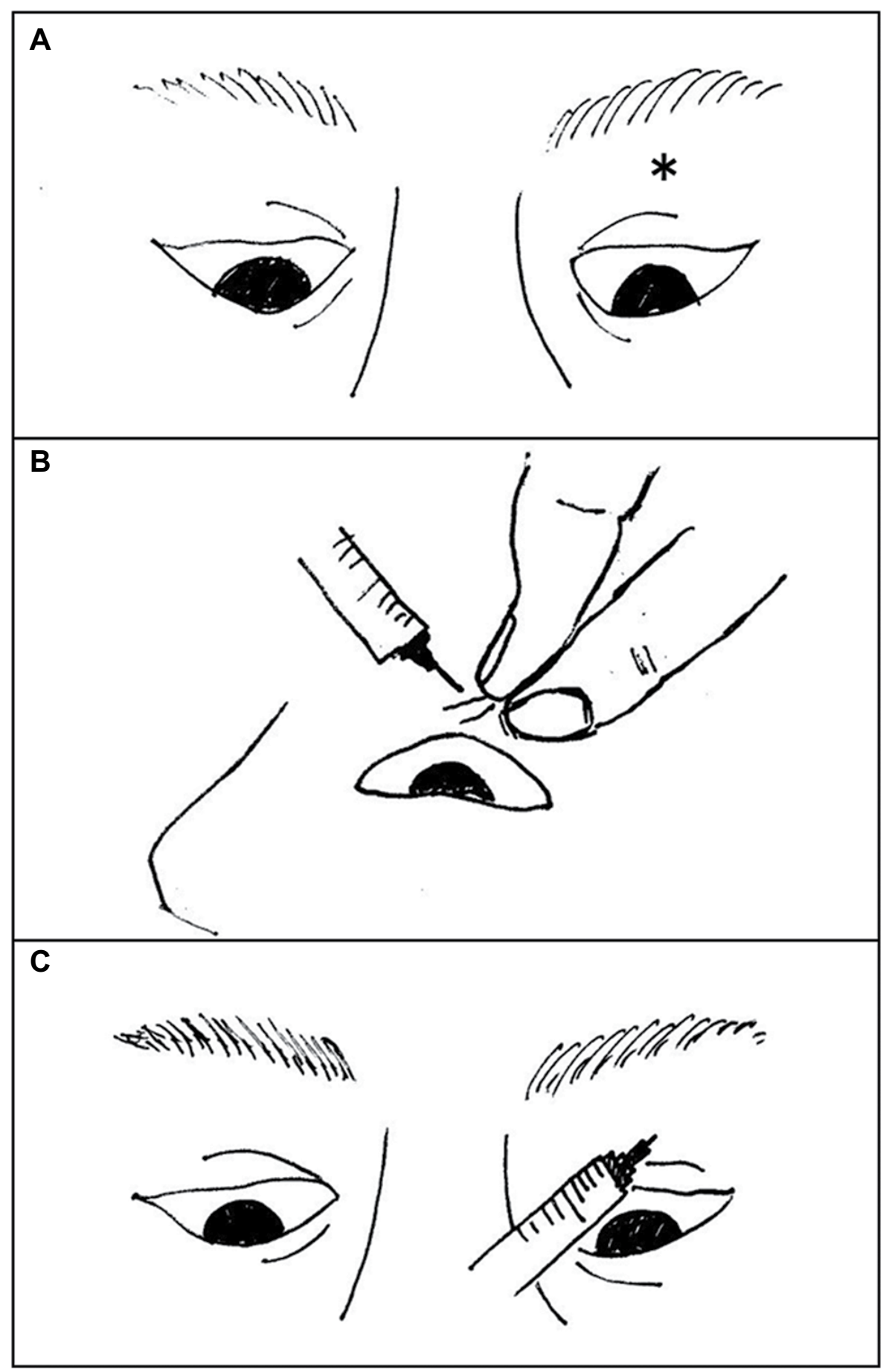

Figure I The skin of the upper eyelid was pulled upward under downward gaze in the supine position).

Notes: The needle injection site $\left(^{*}\right)$ was the center of the eyelid sulcus (A). A 26-guage needle was inserted slightly laterally to an approximate depth of I $\mathrm{cm}$ from the surface of the skin (B). Then, $0.5 \mathrm{~mL}$ of triamcinolone acetonide $(40 \mathrm{mg} / \mathrm{mL}$ ) was gradually injected after confirming the absence of blood reflux (C).

\section{Results}

\section{Case I}

A 35-year-old male TED patient presented with right upper eyelid retraction and swelling for 3 months. His thyroid function was normal without any medication, and he was positive for antithyroid autoantibodies, that is, TRAb ( $\geq 2.0 \mathrm{IU} / \mathrm{L})$ and TSAb (>180\%), as shown in Table 1. He was a nonsmoker and did not receive radioiodine $(\mathrm{RI})$ therapy. At the first visit, palpebral fissure height was 11 and $9 \mathrm{~mm}$ in the right and left eye, respectively. Scleral show, bulging eyelid and a lack of the 
upper eyelid sulcus were observed in the right eye (Figure 2). No proptosis was detected in either eye. MRI taken on the same day revealed LPS muscle enlargement and fat swelling. Prolonged T2 relaxation time and high-intensity areas in T2-weighted fat-suppression images were also noted in the LPS muscle, indicating active inflammatory edema in the muscle (Figure 3; Table 2). Transcutaneous TA was injected in the right eyelid on the same day without any complication.

At 1 month after treatment, the palpebral fissure height reduced and an upper eyelid sulcus was slightly detected. At 6 months, scleral show was absent and the palpebral fissure height reduced to $9 \mathrm{~mm}$, which was the same as the contralateral eye. There was no eyelid bulging, and the upper eyelid sulcus was obvious. At 12 months, no features of upper eyelid retraction or swelling were observed. In terms of post-TAinjective complication, particles of TA (Figure 2) were subcutaneously observed at 1, 3 and 6 months, whereas they were not detected immediately after injection and at 12 months. On MRI taken at 8 months, the LPS muscle and ROOF were clearly thinner than those in images before treatment.

Table I Clinical characteristics

\begin{tabular}{|l|l|l|l|}
\hline Case & I & $\mathbf{2}$ & $\mathbf{3}$ \\
\hline Age, years & 35 & 46 & 48 \\
Gender & Male & Female & Female \\
Smoking & N/A & N/A & N/A \\
FT4 & 1.0 & 1.38 & 1.24 \\
TSH & 0.393 & 0.88 & 0.63 \\
TRAb & 3.2 & 2.2 & 11.7 \\
TSAb & 219 & 296 & 875 \\
Antithyroid drug & N/A & N/A & Propylthiouracil \\
Radioiodine therapy & N/A & N/A & N/A \\
\hline
\end{tabular}

Notes: In all cases, FT4 and TSH were in normal range, while TRAb and TSAb showed an increase. Normal ranges: FT4 $0.90-1.70 \mathrm{ng} / \mathrm{dL}, \mathrm{TSH} 0.50-5.0 \mu \mathrm{lU} / \mathrm{mL}$, TRAb $<2.0$ IU/L, TSAb $<180$.

Abbreviations: TRAb, TSH receptor antibodies; TSAb, thyroid stimulating antibodies.
In addition, both high-intensity signals on T2-weighted fatsuppressed images and prolonged $\mathrm{T} 2$ relaxation time were normalized at 8 months (Figure 3; Table 2).

\section{Case 2}

A 46-year-old female euthyroid TED patient presented with left upper eyelid retraction and swelling for 5 months. She was positive for antithyroid autoantibodies (Table 1). She was a nonsmoker and did not receive RI therapy. At the first visit, right and left palpebral fissure height was 10 and 12 $\mathrm{mm}$, respectively. Scleral show, eyelid bulging and lack of an upper eyelid sulcus were all noted in the left eye (Figure 2). No proptosis was detected. MRI confirmed LPS muscle enlargement and fat swelling along with inflammatory edema (Figure 3; Table 2). TA was transcutaneously injected at 1 month after the first visit, without any complications.

Improvement was observed at 1 month after treatment, as evidenced by appearance of an eyelid sulcus. At 6 months, scleral show and palpebral fissure height reduced, there was no eyelid bulging, and the eyelid sulcus re-formed. At 12 months, all clinical parameters were similar to those in the contralateral eye (Figure 2). MRI at 8 months confirmed normalization of swelling and inflammatory edema (Figure 3; Table 2).

\section{Case 3}

A 48-year-old female TED patient presented with bilateral eyelid retraction and swelling for 3 months. Her thyroid function was well controlled with $200 \mathrm{mg}$ propylthiouracil per day, and both antithyroid autoantibodies were present (Table 1). She was a nonsmoker and did not receive RI therapy. At the first visit, palpebral fissure height (12 $\mathrm{mm}$ ) and proptosis (19 $\mathrm{mm}$ ) were observed in both eyes. Scleral show, eyelid bulging and lack of an upper eyelid sulcus were noted bilaterally (Figure 2). MRI confirmed bilateral LPS muscle enlargement

Table 2 Palpebral fissure height and T2 relaxation time before and after TA injection

\begin{tabular}{|l|l|l|l|}
\hline Case & I Right/left & 2 Right/left & 3 Right/left \\
\hline Palpebral fissure height $(\mathrm{mm})$ & & & \\
$\quad$ Before treatment & $11 / 9$ & $10 / 12$ & $12 / 12$ \\
I month after treatment & $10 / 9$ & $10 / 12$ & $11 / 12$ \\
3 months after treatment & $10 / 9$ & $10 / 11$ & $10 / 11$ \\
6 months after treatment & $9 / 9$ & $10 / 11$ & $10 / 10$ \\
I2 months after treatment & $9 / 9$ & $10 / 10$ & $10 / 10$ \\
T2 relaxation time (ms) & & & \\
Before treatment & $60.79 / 50.83$ & $51.87 / 76.68$ & $72.80 / 78.89$ \\
6-8 months after treatment & $46.26 / 45.70$ & $45.92 / 47.04$ & $46.37 / 51.68$ \\
\hline
\end{tabular}

Note: Right eye was treated in case I, left eye in case 2, and both eyes were treated in case 3. Abbreviation: TA, triamcinolone acetonide. 


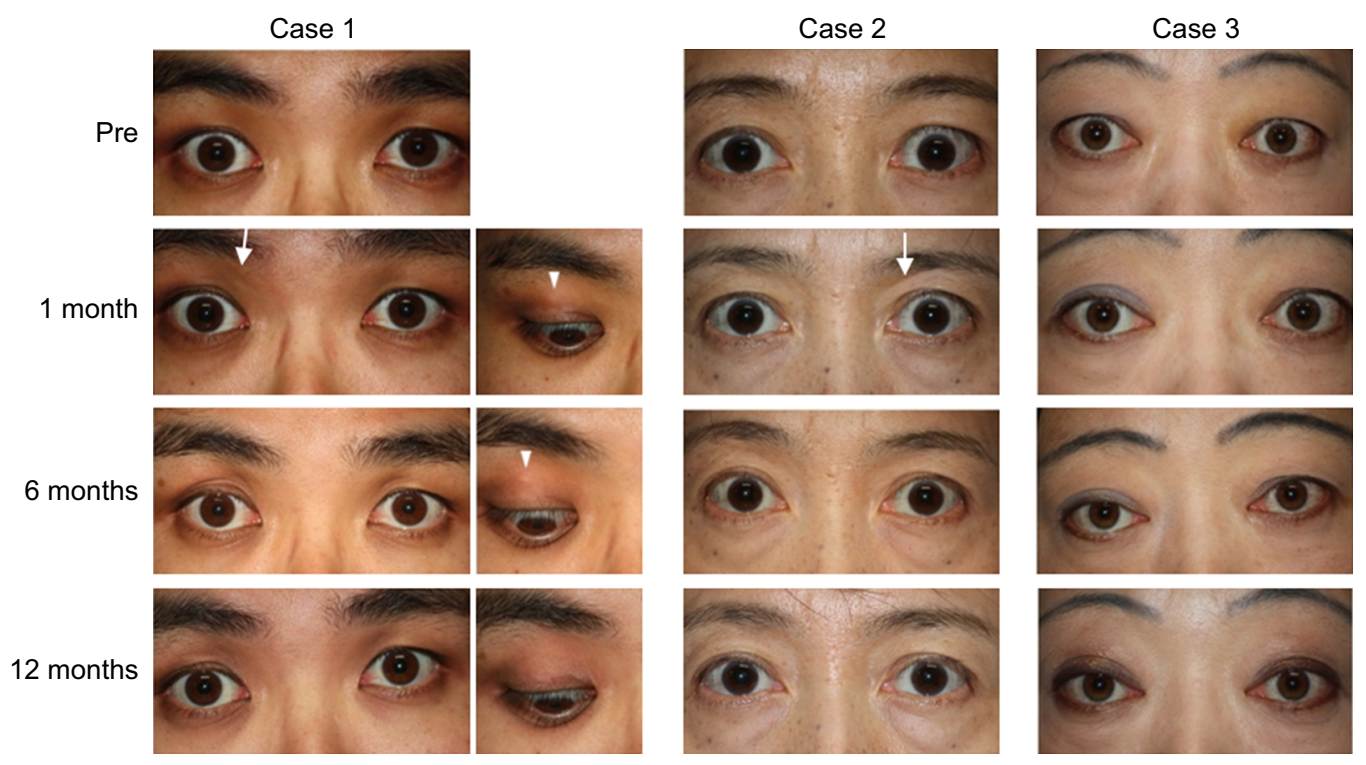

Figure 2 Case I: The right eye was treated. Before treatment, upper eyelid swelling, scleral show and a lack of an upper eyelid sulcus were evident in the right eye. Upper eyelid sulcus (arrow) slightly appeared I month after the injection. At 6 months, the scleral show and eyelid bulge resolved. At 12 months, no features of upper eyelid retraction and swelling were observed. Subcutaneous white particles of TA (arrowhead) were observed at I and 6 months. These were not detectable right after injection. The particles were not visible at 12 months. Case 2: The left eye was treated. Before treatment, scleral show, upper eyelid swelling and lack of an upper eyelid sulcus were evident in the left eye. At I month after treatment, a slight upper eyelid sulcus (arrow) could be detected. At 6 months, the scleral show and palpebral fissure height reduced. At 12 months, the scleral show resolved. Case 3: Both eyes were treated. Before treatment, scleral show, bulging eyelids and lack of an upper eyelid sulcus were evident in both eyes. At I month after treatment, the eyelid bulge reduced. At 6 months, the scleral show resolved and the palpebral fissure height reduced. At 12 months, the eyelid bulge resolved, although the upper eyelid sulcus was still not observable.

Abbreviation: TA, triamcinolone acetonide.
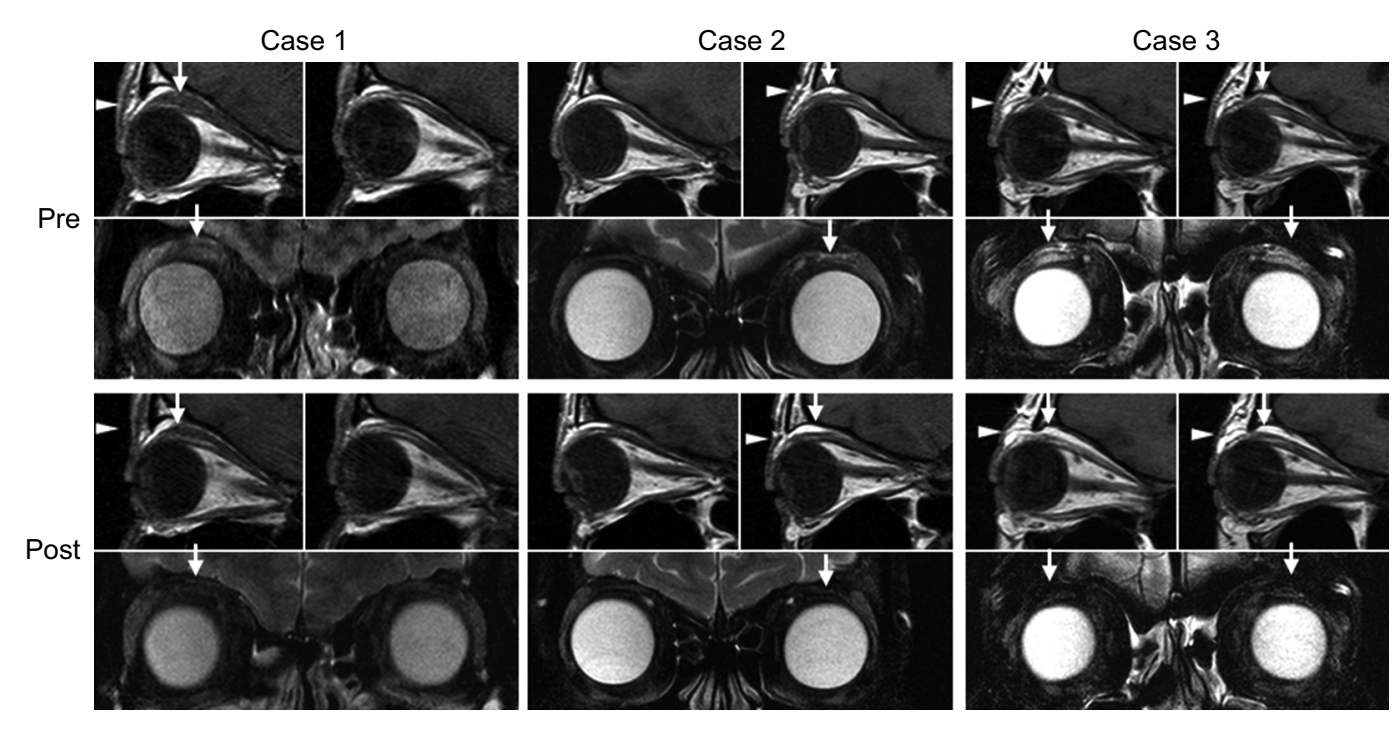

Figure 3 Case I: Pretreatment: The top left image shows TI-weighted MRI sagittal sections. Enlargement of the LPS muscle (arrow) and swelling of eyelid fat (arrowhead) are observable in the right eye, but not in the left eye (top right). Bottom image shows high-intensity signal in the LPS muscle (arrow) on a T2-weighted fat-suppressed image. Eight months posttreatment: Top left image shows that enlargement of the LPS muscle (arrow) was reduced and swelling of fat tissue (arrowhead) was not apparent. Bottom image shows that high-intensity signal in the LPS muscle (arrow) disappeared. Case 2: Pretreatment: Top right image shows mild enlargement of the LPS muscle (arrow) and mild swelling of eyelid fat (arrowhead) in the left eye. Bottom images show high-intensity signal in the LPS muscle (arrow) on a T2-weighted fat-suppressed image. Eight months posttreatment: Top right image shows that the LPS muscle (arrow) enlargement tended to improve slightly and fat tissue (arrowhead) swelling was not observable. Bottom image shows that high-intensity signal in the LPS muscle (arrow) disappeared. Case 3: Pretreatment: Top image shows enlargement of the LPS muscle (arrow) and swelling of fat tissue (arrowhead) in both eyes. Bottom shows high-intensity signals in the LPS muscle (arrow) on T2-weighted fat-suppressed image. Six months posttreatment: Top image shows improvement of enlargement of the LPS muscle (arrow) and swelling of fat tissue (arrowhead). Bottom image shows that high-intensity signals in the LPS muscle (arrow) disappeared on a T2-weighted fat-suppressed image.

Abbreviation: LPS, levator palpebrae superioris. 
and fat swelling, along with inflammatory edema (Figure 3; Table 2). Transcutaneous TA was injected in both eyelids at 1 week after the first visit, without any complications.

At 1 month after TA injection, bilateral eyelid bulging was reduced. At 6 months, scleral show completely disappeared and palpebral fissure height reduced. At 12 months, palpebral fissure height was $10 \mathrm{~mm}$ in both eyes and eyelid bulging had resolved. An upper eyelid sulcus, however, was still not observable in either eye (Figure 2). MRI at 6 months revealed improvement, as indicated by a thinner LPS muscle and less ROOF, along with normalization of inflammatory edema.

In all cases, thyroid function was well controlled during the follow-up period. In addition, no adverse effects arising from TA injection, for example, pigmentation and menstrual disorder, and no recurrence of eyelid retraction or swelling were detected.

\section{Discussion}

To reduce inflammation in the LPS muscle and eyelid fat, TA was transcutaneously injected targeting the orbital fat around the LPS muscle in three TED patients with eyelid retraction and swelling. In all cases, treatment led to resolution of upper eyelid retraction and swelling. Improvement of eyelid retraction was confirmed using MRI, with reduction of LPS muscle enlargement associated with a decrease of inflammatory edema, as evidenced by less LPS high-intensity signal on T2-weighted fat-suppressed images and normalization of T2 relaxation time. ${ }^{16}$ In terms of eyelid swelling, improvement was detected at 1 month after treatment in all cases and an eyelid sulcus reappeared in two cases eventually. Reduced eyelid fat swelling was observed in all cases on MRI. Less improvement, however, was observed in case 3, as an eyelid sulcus was not observable up to 12 months after treatment. As case 3 was the most severe, being the only case with proptosis and having higher TRAb and TSAb than others, ${ }^{25,26}$ this phenomenon may have affected the amount of improvement in eyelid swelling.

In a previous report, ${ }^{23}$ reduction of LPS muscle enlargement was detected after subconjunctival TA injection on MRI, consistent with resolution of inflammatory edema in the LPS muscle and eyelid fat. In this study, a similar improvement in inflammatory edema was confirmed with our MRI findings, including T2-weighted fat-suppressed images and normalization of $\mathrm{T} 2$ relaxation time. Our patients were treated with single transcutaneous TA injection, which is considered an easier administration of the drug to the target tissue, that is, the LPS muscle and eyelid fat in these cases. Our single transcutaneous TA injection demonstrated similar efficacy to single, as well as multiple, subconjunctival TA injection, ${ }^{21-24}$ perhaps with fewer adverse reactions. It is thought that transcutaneous TA injection provides a better approach to target the LPS muscle and surrounding fat tissues.

No adverse reactions following subconjunctival TA injection were noted, although small subcutaneous nodule was noted in one case. In this case, the injection may have been shallower, resulting in TA particles becoming visible through the skin as eyelid fat swelling reduced. These visible white particles, however, were not cosmetically obvious and completely disappeared by the 12-month assessment point.

We found in this study that transcutaneous TA injection was clinically effective for upper eyelid retraction and swelling in euthyroid TED patients when treatment was initiated within 6 months of the appearance of TED signs. Thus, transcutaneous TA injection may be a promising firstline treatment for upper eyelid retraction and swelling with inflammation of the LPS muscle in euthyroid TED patients. However, our sample size was small, and further studies with larger sizes are needed to validate the efficacy of transcutaneous TA injection.

\section{Acknowledgment}

The authors would like to thank R Inoue, G Ito, C Funaki, N Yaji and K Nishiyama for useful discussions.

\section{Author contributions}

All authors contributed toward data analysis, drafting and revising the paper and agree to be accountable for all aspects of the work. All authors read and approved the final manuscript.

\section{Disclosure}

The authors report no conflicts of interest in this work.

\section{References}

1. Bartley GB, Fatourechi V, Kadrmas EF, et al. Clinical features of Graves' ophthalmopathy in an incidence cohort. Am J Ophthalmol. 1996;121(3):284-290.

2. Kozaki A, Inoue R, Komoto N, et al. Proptosis in dysthyroid ophthalmopathy: a case series of 10,931 Japanese cases. Optom Vis Sci. 2010;87(3):200-204.

3. Dickinson AJ. Clinical Manifestations. Graves' orbitopathy: a multidisciplinary approach questions and answers. Wiersinga WM,Kahaly GJ, editors. Basel: Karger;2010:1-25.

4. Daumerie C. Epidemiology. Graves'orbitopathy: a multidisciplinary approach questions and answers. Wiersinga WM,Kahaly GJ, editors. Basel: Karger;2010:33-9.

5. Ponto KA, Pitz S, Pfeiffer N, et al. Quality of life and occupational disability in endocrine orbitopathy. Dtsch Arztebl Int. 2009;106(17):283-289.

6. Terwee CB, Gerding MN, Dekker FW, Prummel MF, Wiersinga WM. Development of a disease specific quality of life questionnaire for patients with Graves' ophthalmopathy: the GO-QOL. Br JOphthalmol. 1998;82(7):773-779. 
7. Duke-Elder S, MacFaul PA. Motor disorders and deformations of the lids. In: System of Ophthalmology. Vol. XIII, part I, chap. VIII. London: Henry Kimpton; 1974:568-570.

8. Mourits MP. Historical Note on Graves' disease. Graves' orbitopathy: a multidisciplinary approach questions and answers. Wiersinga WM,Kahaly GJ, editors. Basel: Karger;2010:271-9.

9. Gay AJ, Wolkstein MA. Topical guanethidine therapy for endocrine lid retraction. Arch Ophthalmol. 1966;76(3):364-367.

10. Ohnishi T, Noguchi S, Murakami N, et al. Levator palpebrae superioris muscle: MR evaluation of enlargement as a cause of upper eyelid retraction in Graves disease. Radiology. 1993;188(1):115-118.

11. Smith TJ, Hegedüs L. Graves' disease. $N$ Engl J Med. 2016;375(16): $1552-1565$.

12. Hiromatsu Y, Sato M, Inoue Y, et al. Localization and clinical significance of thyrotropin receptor $\mathrm{mRNA}$ expression in orbital fat and eye muscle tissues from patients with thyroid-associated ophthalmopathy. Thyroid. 1996;6(6):553-562.

13. Bartalena L, Baldeschi L, Dickinson A, et al. Consensus statement of the European Group on Graves' orbitopathy (EUGOGO) on management of GO. Eur J Endocrinol. 2008;158(3):273-285.

14. Inoue Y, Higashide T, Yoshikawa K, Inoue T. Sagittal magnetic resonance imaging of dysthyroid ophthalmopathy. Eur J Ophthalmol. 1993;3(1):31-36.

15. Ohnishi T, Noguchi S, Murakami N, et al. Extraocular muscles in Graves ophthalmopathy: usefulness of $\mathrm{T} 2$ relaxation time measurements. Radiology. 1994;190(3):857-862.

16. Hiromatsu Y, Kojima K, Ishisaka N, et al. Role of magnetic resonance imaging in thyroid-associated ophthalmopathy: its predictive value for therapeutic outcome of immunosuppressive therapy. Thyroid 1992;2(4):299-305.
17. Ismailova DS, Fedorov AA, Grusha YO. Ocular surface changes in thyroid eye disease. Orbit. 2013;32(2):87-90.

18. Shih MJ, Liao SL, Lu HY, Hy L. A single transcutaneous injection with Botox for dysthyroid lid retraction. Eye. 2004;18(5):466-469.

19. Zang S, Ponto KA, Kahaly GJ. Clinical review: intravenous glucocorticoids for Graves' orbitopathy: efficacy and morbidity. J Clin Endocrinol Metab. 2011;96(2):320-332.

20. Kazim M, Gold KG. A review of surgical techniques to correct upper eyelid retraction associated with thyroid eye disease. Curr Opin Ophthalmol. 2011;22(5):391-393.

21. Chee E, Chee SP. Subconjunctival injection of triamcinolone in the treatment of lid retraction of patients with thyroid eye disease: a case series. Eye. 2008;22(2):311-315.

22. Lee JM, Lee H, Park M, Baek S. Subconjunctival injection of triamcinolone for the treatment of upper lid retraction associated with thyroid eye disease. J Craniofac Surg. 2012;23(6):1755-1758.

23. Xu D, Liu Y, Xu H, Li H. Repeated triamcinolone acetonide injection in the treatment of upper-lid retraction in patients with thyroid-associated ophthalmopathy. Can J Ophthalmol. 2012;47(1):34-41.

24. Lee SJ, Rim TH, Jang SY, et al. Treatment of upper eyelid retraction related to thyroid-associated ophthalmopathy using subconjunctival triamcinolone injections. Graefes Arch Clin Exp Ophthalmol. 2013;251(1):261-270.

25. Eckstein AK, Plicht M, Lax H, et al. Thyrotropin receptor autoantibodies are independent risk factors for Graves' ophthalmopathy and help to predict severity and outcome of the disease. J Clin Endocrinol Metab. 2006;91(9):3464-3470.

26. Mukasa K, Yoshimura Noh J, Kouzaki A, et al. TSH receptor antibody titers measured with a third-generation assay did not reflect the activity of Graves' ophthalmopathy in untreated Japanese Graves' disease patients. Endocr J. 2016;63(2):151-157.
International Medical Case Reports Journal

\section{Publish your work in this journal}

The International Medical Case Reports Journal is an international, peer-reviewed open-access journal publishing original case reports from all medical specialties. Previously unpublished medical posters are also accepted relating to any area of clinical or preclinical science. Submissions should not normally exceed 2,000 words or

\section{Dovepress}

4 published pages including figures, diagrams and references. The manuscript management system is completely online and includes a very quick and fair peer-review system, which is all easy to use. Visit http://www.dovepress.com/testimonials.php to read real quotes from published authors. 